\title{
Téoros
}

Revue de recherche en tourisme

\section{Le tourisme dans l'Est du Québec} L'essentiel reste encore à faire

\section{Gilles Brien, Velitchko Velikov et Jacques Roy}

Volume 5, numéro 1, mars 1986

Tourisme et régions

URI : https://id.erudit.org/iderudit/1080585ar

DOI : https://doi.org/10.7202/1080585ar

Aller au sommaire du numéro

Éditeur(s)

Université du Québec à Montréal

ISSN

0712-8657 (imprimé)

1923-2705 (numérique)

Découvrir la revue

Citer cet article

Brien, G., Velikov, V. \& Roy, J. (1986). Le tourisme dans l'Est du Québec :

l'essentiel reste encore à faire. Téoros, 5(1), 7-8.

https://doi.org/10.7202/1080585ar d'utilisation que vous pouvez consulter en ligne.

https://apropos.erudit.org/fr/usagers/politique-dutilisation/ 


\title{
L'essentiel reste encore à faire Le tourisme dans I'Est du Québec
}

\author{
par Gilles Brien, Velitchko Velikov et Jacques Roy *
}

La croissance économique et le progrès social qui en résulte ne sont pas uniformément répartis dans nos pays occidentaux. Cette question des disparités régionales n'est pas nouvelle; il est généralement admis que nous ne pouvons pas laisser les forces du marché seules responsables du bien-être des citoyens et dans ce sens, l'État s'est vu confier un rổe important dans la redistribution spatiale des bénéfices de notre économie.

\section{Les contradictions du développement régional}

Peut-on espérer "corriger" une situation qui apparaît comme étant au coeur mẻme de notre système, c'est-à-dire la libre confrontation de l'offre el de la demande? Sur le plan spatial nous savons que cela crée une croissance dite déséquilibrée,

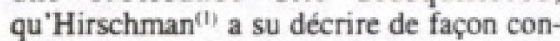
vaincante. Il apparait donc inévitable que les "centres" exercent un effet de polarisation au détriment des "périphéries" qui $y$ perdent ressources, capitaux et maind'oeuvre.

Si nous retenons comme facteur majeur des disparités régionales l'orientation des flux economiques vers la métropole, nous pouvons formuler I'hypothese suivante: il suffit, pour provoquer la croissance économique des périphéries, de réussir à neutraliser les forces centripètes qu'exerce le centre. Mais comment? A notre avis, le tourisme est un secteur d'activités économiques dont la nature mëme se prêtte à cette fin.

\section{Le tourisme: facteur d'équilibre?}

Le produit touristique étant non-exportable, il permet une inversion du modele centrepériphérie. Il favorise l'existence d'une "péréquation spontanée", c'est-à-dire d'un flux de capitaux du centre vers la périphérie.

Globalement, ce phénomène n"est qu"une redistribution lorsque nous parlons d'une

\footnotetext{
"Les trois auteurs sont protesseurs de gégraphie IUniversite du Quebec a Rimouski.
}

clientèle touristique nationale. Mais même dans ce contexte, cette activité économique reste intéressante, et cela pour detux raisons. D'abord, parce que ces placements de transfert ont un effet multiplicateur appréciable; ensuite, parce qu'une augmentation de la performance touristique nationale peut freiner l'exode des vacanciers vers I'étranger.

De plus, Jorsque la clientèle touristique vient de l'extérieur, nous avons alors une activité écnomique d'exportation, ce qui présente un grand intérêt en termes de balance commerciale et de potentiel teonomique national. Ultimement, cet influx de capitaux permet une rentabilisation des infrastructures de développement mises en place par l'État. À ce stade, nous ne parlons plus de disparité mais plutôt de prospérité économique.

Cette vision du développement touristique n'est pas qu'idéalisme. En effet, lorsqué nous regardons les importanies stations touristiques actuelles, nous constatons qu'elles sont souvent, sinon toujours, situées dans des régions antérieurement en difficulté ou en état de sous-développement économiqué. Il suffit, à la suite de Sprincova ${ }^{(2)}$, de se rappeler les exemples de la Cóte d'Azur ou des stations alpines des pays occidentaux ou le cas du littoral de la mer Noire dans les pays de l'Est.

\section{Le prix du développement touristique}

Mais ce développement touristique ne se fait pas seul. Barbaza ${ }^{(3)}$ nous propose trois types d'intervention auxquels correspondent trois types d'organisation de l'espace. Nous ne retiendrons ici que le mode d'intervention qui est soit étatique à l'exemple du développement des littoraux bulgares ou roumains, soit privé, telle l'expérience de la Côte d"Azur ou de la Costa Brava ou, finalement mixte, du type Languedoc-Roussillon. Dans le cas du Québec, il faut constater que l'essentiel du développement économique des régions repose sur l'entreprise privée, à l'exception bien entendu de la mise en place des infrastructures d accompagnement tels, les routes, les aménagements des littoraux, les quais, etc, qui sont nécessaires comme support à l'ensemble des activités socioéconomiques, et la création des parcs el réserves dont la mission est d'abord et avant tout la conservation du patrimoine naturel.

Mais cela pose un problème. En effet, puisque le developpement touristique repose sur l'entreprise privếe. les régions en déclin se voient prises dans un cercle vicieux: pas de développement, pas d'investissement privé: pas d'investissement privé, pas de développement... L'intervention de l'Etat devient done obligatoire; et au Québec, les choses sont claires: nous n'avons pas de plan global de développement touristique, ni de ministère du Tourisme bien défini, ni de volonté réelle de développement régional.

\section{Une activité éclatée}

Bien que le tourisme dans l'Est du Québec ne soit pas un phénomène nouveau, son apport pour le developpement semble toujours mis en doute. Cela s'explique probablement d'abord par le grand éclatement de ce secteur économique. En effet, parler de tourisme c"est parler de réalités aussi diverses que de restauration, hébergement, ali= mentation, cinéma, camping, chasse et péche, artisanat, fettes populaires, transport. etc. Il n'est pas surprenant que la structure organisationnelle correspondante soit anarchique. Nous y retrouvons une multitude d'intervenants de tous les paliers de gou* vernement et de tous les secteurs de l'entreprise privée: MEIR, MIC, MLCP, OPDQ. Chambres de commerce, Conseils municipaux, MRC, ATR, Conseils de loisirs, ete.

Il ne faut donc pas se surprendre de voir que les actions de développement touristique aient toujours été caractérisées, dans l'Est du Québec, par la dispersion spatiale. par la discontinute temporelle el par l'anarchie organisationnelle. Quelques exemples suffisent pour mettre clairement ces faits en évidence.

Notre-Dame-du-Lac, municipalité riveraine du lac Témiscouata, est un centre de villégiature plus que centenaire. Ces derniêres années, des efforts ont été entrepris pour stimuler le secteur touristique et en faire un véritable moteur de développement; toute- 


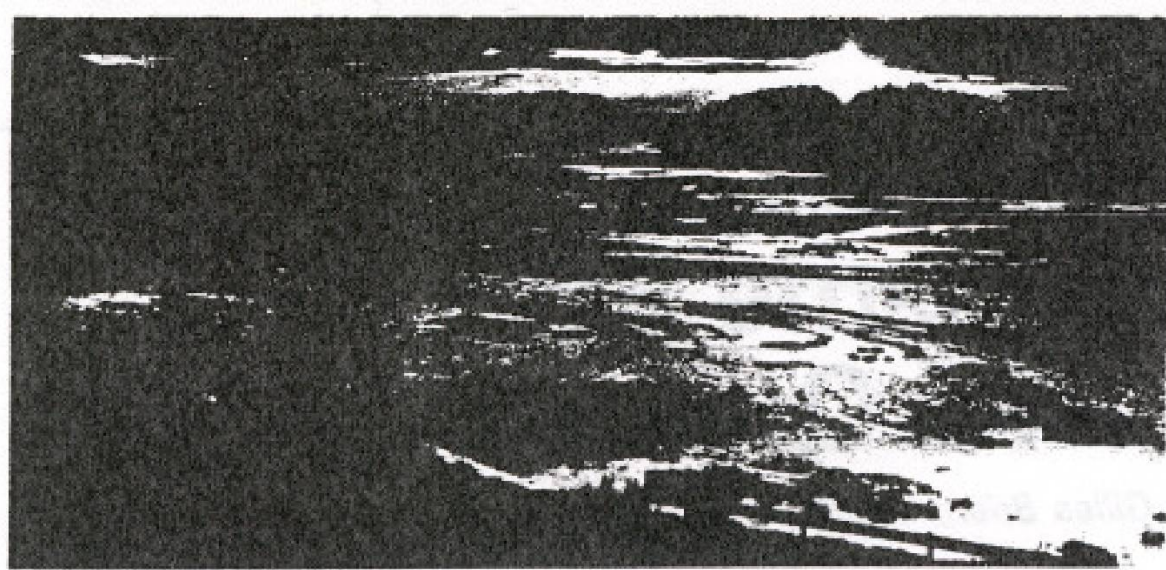

Le Parc du Bic, malgré son indêniable beauté, continue d’ëtre une réalité sans substance

fois, le bilan de ces expériences est loin d'être convaincant. Plusieurs raisons l'expliquent, mais il est clair que le manque d"intégration verticale au niveau de la structure organisationnelle, y est pour beaucoup.

Dans les années '70, le dossier est pris en charge par la municipalité, par le biais d'un organisme local de développement touristique, en collaboration avec le Conseil intermunicipal des loisirs du Témiscouata (CILT), à l'aide de fonds provenant surtout de projets fédéraux de création d'emploi et de ministères provinciaux pour les réalisations sectorielles. A la fin de cette décennie apparait l'Association touristique du Bas-St-Laurent (ATRBSL) et quelques années plus tard la Municipalité régionale du Témiscouata. Tout cela conduit forcément à recommencer continuellement des choses déjà faites, si bien que Notre-Damedu-Lac a maintenant deux plans d aménagement touristique exhausrifs, sans pour autant être témoin d'un véritable démarrage économique. Un autre problème réside dans la difficulté d'intégration hirozontale due à l'absence de volonté politique de développement régional, qui se traduirait par l'existence d"une structure ministérielle nationale et de moyens nécessaires pour piloter un plan de développement touristique québécois.

\section{L'éternel recommencement}

Ce problème de structures organisationnelles est egalement très clair dans le cas de la Vallée de la Matapéria, région caractérisé par sa tradition de chasse et de péche et par sa localisation sur l'itinéraire du "tour de la Gaspésie"t.

A la suite des Opérations Dignité (OD) et gráce à des efforts de quelques personnes. la Société d'exploitation des ressources de la Vallée (SERV), organisme d'aménagement intégré des ressources, est "persua= dé". vers la fin des années 70 , de s'occuper du développement touristique. Mais rapidement et pour des raisons tobs. cures", la SERV realise que le tourisme est un secteur trop difficile à concilier avec l'exploitation forestière, mandat principal qu'elle s'est donnee, et confie le dossier à une nouvelle structure sectorielle; TOURMAT , la société privée ainsi créée, reprend le dossier, produit un plan de développement et met en place des infrastructures. Toutefois, à ce moment intervient la loi 125 et la nouvelle Municipalité régionale de comté revendique. par le biais de son mandat d'aménagement, un dossier devenu important.

Nous assistons donc encore une fois aux effets de l'anarchie organisationnelle et à l'éternel recommencement; le mandat de développement touristique est d'abord l'affaire d'un organisme polyvalent (SERV) pour ensuite devenir sectoriel (TOURMAT) pour redevenir global (MRC); pendant ce temps l'Association touristique de la Gaspesie (ATRG) reste prise entre ces jeux de pouvoirs

\section{Le problème des structures organisationnelles}

Ce problème de structure traduit également la difficulté d'intégration horizonale ou l'impossibilité d'etablir un consensus sur les orientation et les priorités du développement. Cette dimension est très claire dans l'arrière-pays de Rimouski, où l'expérience du Corridor 232 est très révélatrice. L'initiative de développement touristique dans cette région agro-forestière vient des $O D$, dans le but de solidariser une population dispersée sur un immense territoire peu nature différente c'était une région à créer L'appellation "Corridor 232 " esi très significative, Elle reflète d'une part l'absence d'un toponyme pour designer ce territoire et d'autre part l'idée de faire de ce secteur un itinéraire alternatif au littoral pour joindre la Vallée de la Matapedia et la Vallée du Témiscouata. Les intervenants touristiques ont donc commence à utiliser cette expression technique simplement pour pouvoir faire référence à cet espace en utilisant le numéro de la route qui le traverse. Rapidement, profitant des efforts d'animation accompagnant l'élaboration du plan de développement touristique, il fallait trouver un autre nom pour désigner cette région. L'évolution du toponyme met en lumière la discontinuité dans le cheminement du dossier. D'arrière-pays de Rimouski, réalité géographique, on passe à Corridor 232. appeliation technique utipeuplé et confronté à des problemes de lisée par les agents de développement: on se retrouve ensuite avec Chemin-Taché. proposé par les chercheurs de l'UQAR et designant un territoire plus grand: finalement le mandat est confie à la Sociéte d'exploitation des ressources de la Neigette (SERN) qui rebaptise ce territoire HautPays-de-la-Neigette.

Cette derniere intervention conncide avec le sommet économiqué de Rimouski de 1982 qui est, depuls le BAEQ, une des rares manifestations d"une volonte politique de developpement régional. Les résolutions adoptées soulevent alors l'enthousiasme des intervenants touristiques, mais quatre ans plus tard nous ne voyons pas beaucoup de réalisations. Le Centre national d'interprétation maritime est toujours à l'étude, le Parc du Bic continue d'être une réalité sans substance et "Rimouski ville de congrès" n'attire finalement que des appuis municipaux. L'absence d'une volonté nationale de développement régional aura encore une fois fait perdre energies el espoirs.

Pendant ce temps, les Associations touristiques de la Gaspésie et du Bas-St-Laurent mandatent la firme Roche et Associés pour établir un plan de développement touristique régional. Terminée en 1985, cette étude devait permettre une relance de l'activité touristique dans l'ensemble du territoire. Mais en fait ce plan, qui constitue un excellent bilan de la question, ne reflete que trop bien la situation nationale, c'est-à-dire l'absence de planification. d'orientation et évidemment de structures permettant réellement le développement régional et la contribution du tourisme a cette fin. Il est trop tớt pour parler de l'inutilité de cette demarche, mais il est clair que les ATR $n$ " $y$ ont pas trouvé ce qu'elles cherchaient, mais plutốt ce qu'elles savaient déjà.

\section{La dialectique État-Région: une question en suspens}

Si nous voulons vraiment atténuer les dis. parités régionales, évirer que les régions périphériques ne se vident et contribuer au bien-être de la population toute entiere, il faudra tôt ou tard qu "on admette que l'État a un róle important à jouer. Car dans l'Est du Québec le développement touristique laisst seul ne risque pas d'ëtre anarchiqué et destructeur, il est au contraire voué à la disparition graduelle. $\boldsymbol{f}$

\section{Ptifirence:}

11) HIRSCHMAN, Albert 0. The Strategy of Econo mic Development. Yale University Press. 1958. 210 payes

(2) SPAINCOVA. St. Le roursme ef he problime des remiaives en dechn dans les pays indusmels. Studia Geographica 20. Bino, 1971, pages $145-158$

(3) BAABAZA Ywete Trous twase dintervention of tourisme dans fargansatron de l'espace hroral. Annales de gtographie, no 434, juiliet-aoút 1970 . pages $446-469$ 